\title{
SYNERGETIC CONTROL AND SYNCHRONISATION OF CHAOTIC SYSTEMS
}

\author{
Harmas Mohamed N. \\ Electrical Engineering Department, QuERE Lab, University of Setif1, Setif, Algeria
}

\begin{abstract}
Synergetic Control and synchronization of two different chaotic systems is presented in this paper and simulation results are given illustrating the effectiveness of the robust control technique applied. Similar to the sliding mode approach but without damaging chattering, synergetic control is a robust approach which doesn't require linearization and should be suitable for real-time implementation for not requiring a discontinuous term in its control law.
\end{abstract}

\section{KEYWORDS}

Synergetic Control; Chaotic Systems; Synchronisation;

\section{INTRODUCTION}

Chaotic phenomena studied for decades and seem to have been discovered by E.N. Lorentz of the department of meteorology at MIT in 1963 when he published a system of differential equations describing a simplified atmosphere model exhibiting non-periodic steady-state behavior that expresses the essence of turbulence [1]. Butterfly or vague attractors issued from the Lorentz and the Lorentz like systems, obtained from differential system equations (1) seem to be a wellknown signature of chaotic phase portraits as shown in figures Fig.1a, b and c. Chaotic behavior has been reported in many real systems such as power systems, power convertors, biological or mechanical systems. Their specifics besides instability, a high sensitivity to operating points as well as fractals like phase portrait and bifurcations have attracted many researchers to address the challenging issues of chaos control and synchronization of chaotic systems. Among many seminal works one can cite Abed and Varaiya [2], Dobson et al [3]. Harb and Abdel-Jabbar [4] analyzed the stability of voltage collapse and Hopf bifurcation and chaos in electrical power systems. Harb et al [5] analyzed the stability and the bifurcation behavior of a modular peak current-mode controlled DC-DC boost converter in power electronics. Anbukumar et al dealt with sliding mode control of chaos in voltage mode buck converter [6], Harb [7] and Endo [8] analyzed the chaos control of phase-looked loop in communication systems. More recently synchronization of chaotic systems has gained an accrued interest in various domains such as cryptography, secure communication, pattern recognition and nonlinear control systems. Lin et al (9) applied. type-2 fuzzy sliding mode control to synchronize chaotic systems, Liu et al [10] used a PI type synergetic control for the same purpose reducing chattering as well as response time. Synchronization of different fractional systems via active control is studied in [12] and synchronization of fractional order Genesio-Tesi system via active control and sliding mode control is reported in [13]. The phenomenon of system chaotic behavior has been perfectly depicted by classes of mathematical oscillators which reveal the oscillating nature of physical systems under specific initial conditions such as the classical Lorentz, Lù, Chua, Genesio-Tesi, Vander Pol-Duffing and Chen's chaotic systems. Illustrating chaotic behavior, Lorentz system (1) is simulated producing fractals like phase portraits. 


$$
\begin{aligned}
& x=(25 a+10)(y-x) \\
& y=(28-35 a) x-x z+(29 a-1) y \\
& z=x y-(8+a) z / 3
\end{aligned}
$$

pour $a=0.1 ; x(0)=-21 ; y(0)=-9 ; z(0)=12$

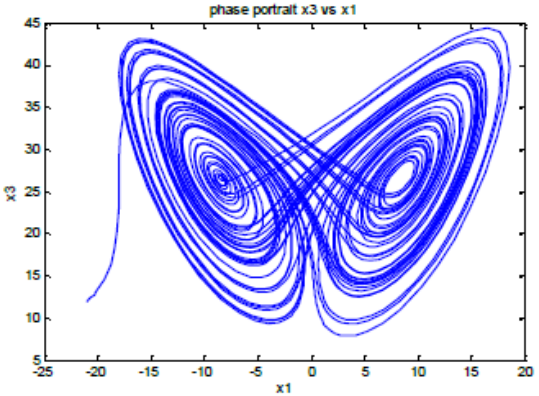

a)

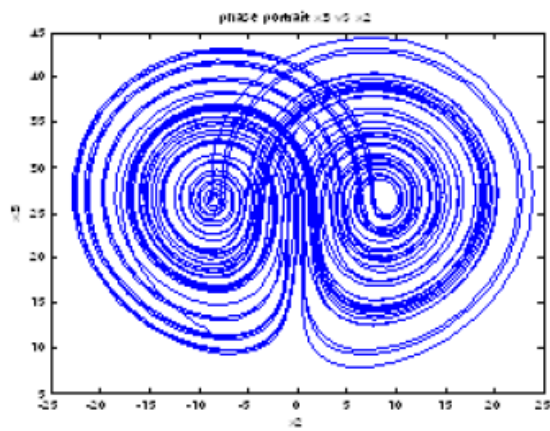

b)

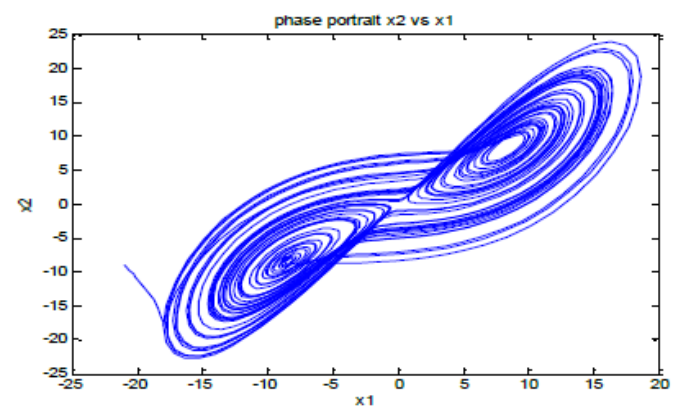

c)

Figure 1 a, b, and c Lorentz chaotic system phase portraits.

The rest of the paper introduces the problem formulation followed by a brief overview of synergetic control. An introduction of the considered chaotic systems ensues followed by simulation and simulation results discussion. A conclusion entailing results finding and perspective work terminates the paper.

\section{SYSTEME DESCRIPTION AND PROBLEM FORMULATION}

\subsection{Chaotic systems}

Chaotic behavior is an unacceptable operating mode which appears for some parameter values and not for others. Non periodic chaotic behavior is surprisingly deterministic thus independent of any external stimulus [1] in chaotic systems. Controlling such systems is a challenging task which has been tackled trough many robust approaches among which sliding mode and synergetic control. The latter will be used to control two chaotic systems without a 
discontinuous control law leading eventually to an ease in implementation.

\subsection{Synchronization of chaotic systems}

The synchronization of two chaotic systems with different initial conditions has been addressed via various approaches. Synergetic control is used in this paper to force a slave chaotic system output to follow the output of a master chaotic systems .Let the master chaotic system be described by (2) and the slave system by (3):

$$
\begin{aligned}
& \lceil x=x \quad 1 \leq i \leq n-1 \\
& \left\{\begin{array}{l}
{ }^{i}=f(x, t) ; \quad x ; x \in R^{n} \\
x_{n}
\end{array}\right. \\
& \text { (n } \quad 0 \\
& \text { \ } y=y \quad 1 \leq i \leq n-1 \\
& \left\{\begin{array}{l}
y . \\
y(y, t)+d(t)+u ; \quad x ; x \in R^{n}
\end{array}\right.
\end{aligned}
$$

where $f(x, t)$ and $g(y, t)$ represent chaotic systems dynamics and $d(t)$ is a bounded disturbance; $\mathrm{u}(\mathrm{t})$ is the synchronizing control while $x_{i}$ and $y_{i}$ designate systems state and outputs. Synchronization consists in elaborating a control law such that both chaotic system outputs are identical despite the difference in nature and initial conditions. Letting the synchronization errors between master and slave be given as:

$$
\begin{aligned}
& e_{i}=y_{i}-x \quad ; \quad 1 \leq i \leq n \\
& e_{i}=e_{i+1} \quad ; 1 \leq i \leq n-1 \\
& e_{n}=g(y, t)-f(x, t)+d(t)+u
\end{aligned}
$$

Error dynamics are then imposed by choosing an appropriate macro-variable or attractor that which in its turn is forced by an evolution constraint to reach the desired equilibrium point. The approach described, similar to the sliding mode approach, but without undesirable chattering, is called synergetic control and is briefly recalled in the next section.

\section{SYNERGETIC CONTROL OVERVIEW}

Synergetic control [10-11] has been around for a few decades and is acquiring rapid recognition by industrials and by the robust control community as well. Practical implementation is illustrated in power electronics [15-17] and in industrial battery charging [18]. Similar to sliding mode methodology, synergetic control is a robust technique with a continuous control law

Therefore without chattering.

Basics of synergetic control synthesis for a nonlinear dynamic system described by (5) are introduced next.

$$
\frac{d x(t)}{x}=f(x, u, t)
$$


where $x$ represents the system state space vector and $u$ its control. Control synthesis begins by a suitable choice of a macro-variable function as in (6):

$\psi=\psi(x, t)$

Where $\psi$ and $\psi(x, t)$ designate designer chosen macro-variable and a corresponding a state Variables and time dependent function. Next a desirable manifold (7) is chosen on which the system will be forced to remain even in presence of unwanted disturbances or parameters fluctuations just as on a sliding mode surface.

$$
\psi=0
$$

Selecting macro-variable features can be realized through many possibilities accordingly with performance objectives and practical physical constraints.

A macro-variable which can be a classic linear combination, is forced to evolve accordingly to the designer imposed constraint as in (8):

$$
T \psi+\psi=0
$$

Speed of convergence depends on selected parameter $\mathrm{T}$ while state trajectories are driven towards the attractor given by (7).

Synergetic control law is obtained via the following steps:

$$
\frac{d \psi(x, t)}{d t}=\frac{d \psi(x, t)}{d x} \cdot \frac{d x}{d t}
$$

Using (5) and (6) in (8) leads to (10):

$$
T \frac{d \psi(x, t)}{d x} f(x, u, t)+\psi(x, t)=0
$$

Resolving (10) for $u$ gives the control law as:

$$
u=g(x, \psi(x, t), T, t)
$$

Synergetic control law u depends not only on system variables but on parameter $\mathrm{T}$ and macrovariable $\psi_{\text {as well, enabling the designer to choose controller performance on a nonlinearized }}$ model. Macro-variables appropriate choice and astute manifolds enforce closed-loop overall stability and invariance to parameter variations [10] for when the system attains the specified manifold it remains on it.

\section{Simulation EXamples}


Consider the two chaotic uncertain systems [9], slave (12) and master (13), for which synergetic control is developped to stabilize both systems separately then synchronization via the same technique will be presented. Simulation of chaotic behavior will be illustrated for both autonomous and synergetic controlled systems.

\subsection{Synergetic control of chaotic systems}

The master system is given as (12):

$$
\begin{aligned}
& x_{1}=x_{2} \\
& \begin{array}{rrrr}
x & =-0.4 x & +1.1 x-x^{3} & \\
2 & 2 & 1 & 1
\end{array}
\end{aligned}
$$

and the slave system equations are given by (13).

$$
\begin{aligned}
& y_{1}=y_{2} \\
& y_{2}=\begin{array}{lll}
3 y-0 . y^{3} & -y^{3} & -1.1 \cos (0.4 t) ; y(0)=[1 ; 1]
\end{array}
\end{aligned}
$$

Selecting macro-variable (14) for system (12) as :

$$
\Psi_{1}=x_{1}+k_{1} x_{1}
$$

and the constraint (15):

$$
T_{1} \Psi_{1}+\Psi=0
$$

where $\mathrm{k}_{1}$ and $\mathrm{T}_{1}$ are designer chosen parameters.

The same procedure is repeated for system (12) and simulation results are given in Fig.2 and Fig.3 for chaotic behaviour and in Fig.4 for synergetic controlled master system (12).

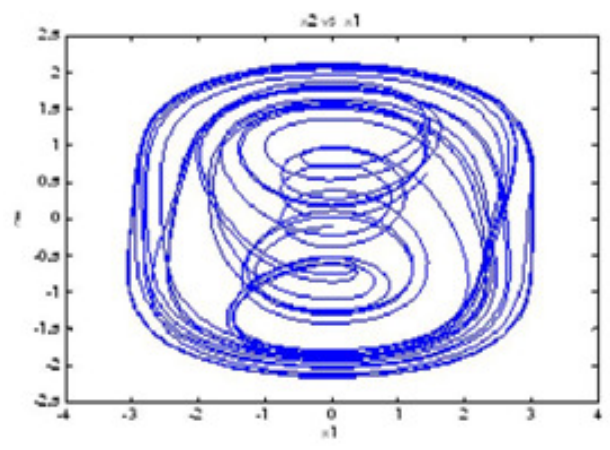

Figure 2. Phase portrait of master system

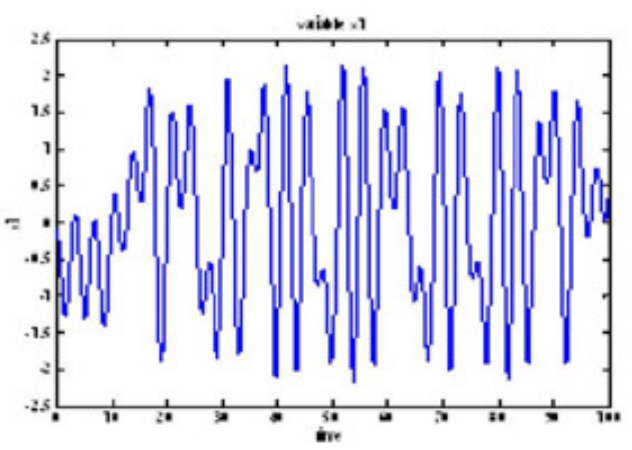

Figure 3. Master chaotic system output. 

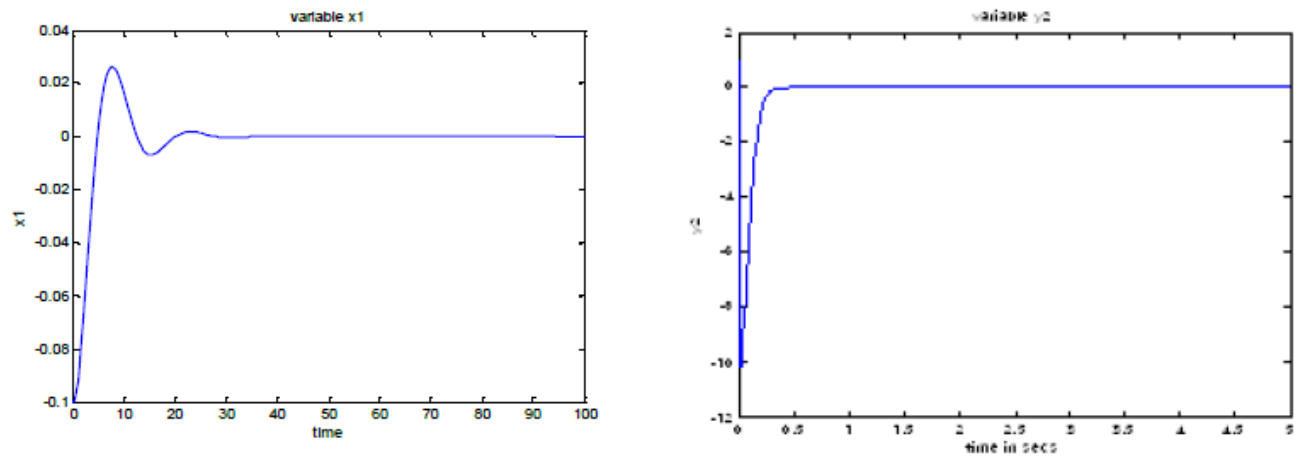

Figure 4. Master system stabilized trajectories

The second system (13) chaotic phase portrait and output are shown on Fig.5 and Fig.6 respectively while Fig.7 represents its stabilized outputs.

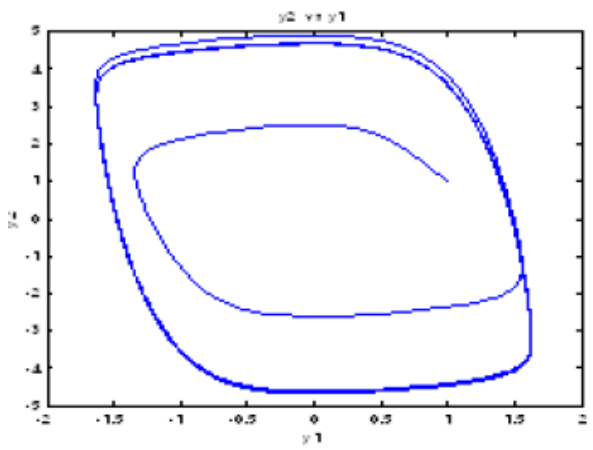

Figure 5. Slave system phase portrait

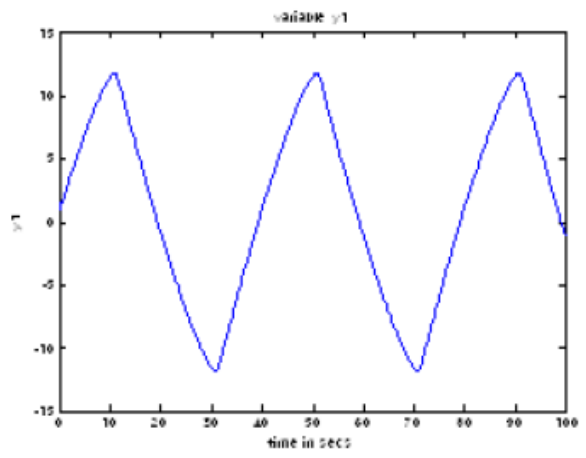

Figure 6. Slave system chaotic output 
International Journal of Control, Automation, Communication and Systems (IJCACS), Vol.1, No.2, April 2016
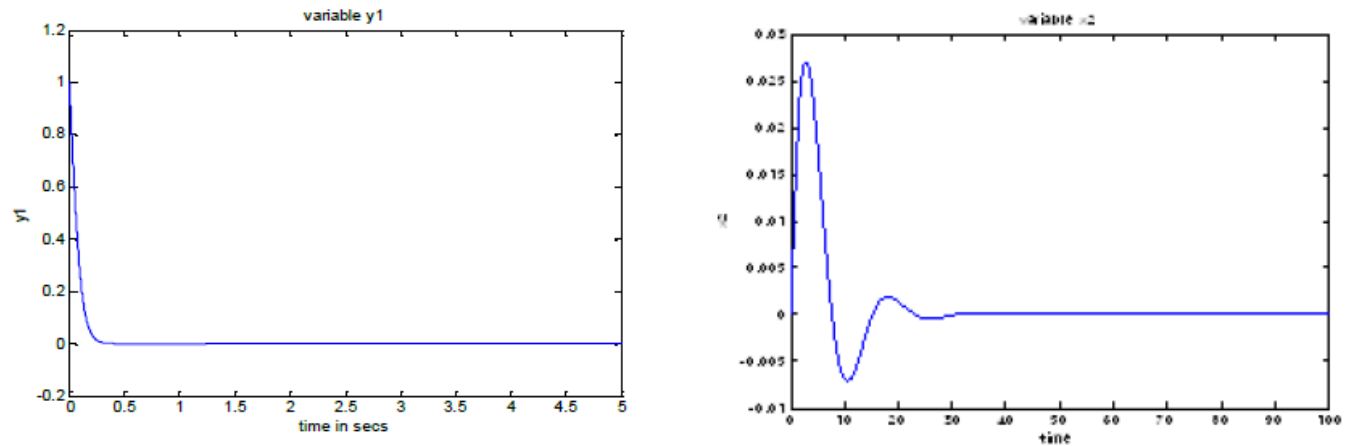

Figure7. Slave system stabilized trajectories 


\subsection{Synergetic synchronization of the two chaotic systems}

It is now desired to synchronize the two chaotic systems using the synergetic approach. System (13) being selected as the slave system will be modified as to include synergetic control $u(t)$ elaborated using the same procedure as before to the new augmented system which will include both systems as in (16). Eq.17 represents the new macro-variable which includes the error between the master and slave systems while a classic constraint (18) similar to previous one is chosen.

$$
\begin{aligned}
& x_{1}=x_{2} \\
& \dot{x}=-\left.0.4\right|_{2} ^{x} \begin{array}{c}
+1.1 x-x^{3}-2.1 \cos (1.8 t) ; x(0)=[-0.1 ; 0] \\
1
\end{array} \\
& y_{1}=y_{2} \\
& y_{2}=1.8 y-0 . y_{2}-y_{1}^{3}-1.1 \cos (0.4 t)+u(t) ; y(0)=[1 ; 1]
\end{aligned}
$$

The synchronization error e is chosen as: $e=y_{1}-x_{1}$ and the new macro-variable as:

$$
\Psi=k e+e=k\left(y_{1}-x_{1}\right)+\left(y_{1}-x_{1}\right)
$$

and the new constraint as:

$$
T \Psi+\Psi=0
$$

Straightforward steps lead to the synergetic control law and simulation results are embodied by figure (8) which indicates a rapid synchronization upholding chaotic behaviour of the systems despite the difference in initial conditions.

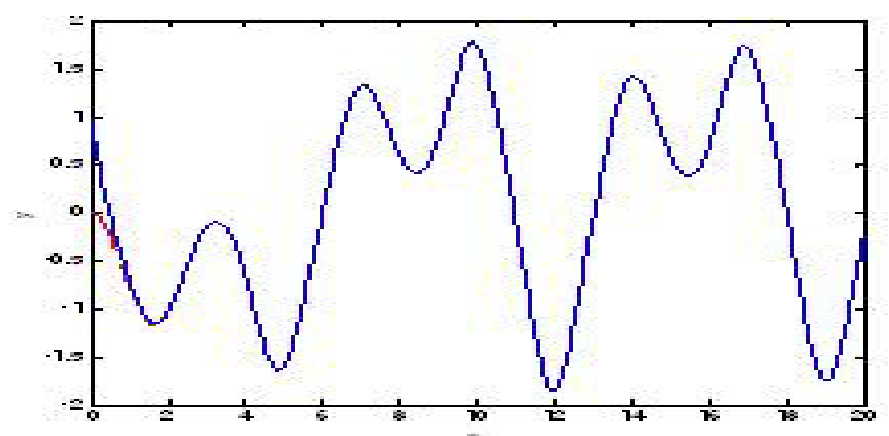

Figure 8. Synchronized chaotic slave-master outputs

One can observe that despite different initials conditions the two systems are rapidly synchronised using synergetic control while staying in their chaotic states that which could eventually be used in securing data transmission.

\section{CONCLUSiON}

Control of two chaotic systems has been adequately achieved, through simulation, using 
synergetic approach. Furthermore their synchronization has been realized despite their different initial conditions. The approach is as robust as sliding mode methodology but relies heavily on model knowledge and on a non-systematic choice of control parameters. These issues are to be tackled by intelligent techniques like the fuzzy approach and controller parameters are to be optimized via evolutionary algorithms.

\section{REFERENCES}

[1] R.W. Brockett, and J.R. Wood, "Understanding power convertor chaotic behaviour mechanisms in protective and abnormal modes", Proc.of Powercon 11, paper E-4, Power Concepts Inc., Ventura, CA, 1984

[2] E.H. Abed, and P.P. Varaiya," Nonlinear oscillations in power systems”, Int.J. Elec. Power Energy Syst. 1989; 6: 37-43.

[3] I. Dobson etal., "A model of voltage collapse in electric power systems". In: IEEE Proc. 27th Conf. on Decision and Control, Austin, TX; 1988. p. 2104-09.

[4] A.M. Harb, N. Abdel-Jabbar, "Controlling Hopf bifurcation and chaos in a small power system", Chaos, Solitons \& Fractals 2003; 18(5):1055-63.

[5] A. Harb ,M. Mothafer, A. Natsheh, "Application of bifurcation theory to current mode controlled parallel-connected DC-DC boost converters", Int. J. M odel Simulation. 2005,25(1).

[6] K. Anbukumar, and U. Govindarajan, "Sliding mode control of chaos in voltage mode buck converter", JEE, vol.11, ed.2,2011, pp.142-152.

[7] Harb B, Harb A. "Chaos and bifurcation in a third-order phase locked loop", Chaos, Solitons \& Fractals 2004;19(3):667-72.

[8] T. Endo , and L.O. Chua, "Chaos from phase-locked loops", IEEE Trans. Circ. Syst. 88;35: 9871003.

[9] T. Lin, M. Chen, and M. Roopeai, "Synchronization of uncertain chaotic systems based on adaptive type-2 fuzzy sliding mode control”, Eng. App. of Art. Int., Elsevier, 2011,vol.24, pp. 39-49.

[10] C.H. Liu, and M.Y. Hsiao, "Synchronization on unified chaotic systems via PI-type terminal synergetic control”, CACS2013, Sun Moon Lake, Taiwan, 2013.

[11] L.Medjbeur, M.N. Harmas, and S. Benaggoune, "Nonsingular terminal synergetic control of induction motor", JEE Vol.16/2016, Ed.1, Art.47.

[12] S. Bhalekar, .D.Gejji, "Synchronization of different fractional order chaotic systems using active control", Commun. Nonlinear Sci. .Numer. Simul.15 (2010)3536-3546.

[13] M.R. Faieghi, H.Delavari, "Chaos in fractional-order Genesio-Tesi system and its synchronization", Commun.Nonlinear Sci.Numer. Simul.17(2012) 731-741.

[14] A. Harb , B. Harb, "Chaos control of a third-order phase locked loop using backstepping nonlinear controller", Chaos, Solitons \&Fractals 2004;20(3):719-23.

[15] A. E .Matouk, "Chaos feedback control and synchronization of a fractional-order modified Autonomous VanderPol-Duffing circuit", Com. Nonlinear Sci. Numer. Simul. 16(2011) : 975- 986.

[16] S. Bhalekar, and D. Gejji, "Synchronization of different fractional order chaotic systems using active control”, Commun. Nonlinear Sci. .Numer. Simul.15 (2010)3536-3546.

[17] D. S. Young, Won Heo , E. Santi , A. Monti, "Synergetic Control Approach for Induction Motor Speed Control", The 30th Conf. IEEE Ind. EIectronics Society, Nov. 2 - 6,2004, Busan, Korea.

[18] A. Monti, E. Santi, K. Proddutur, R.A. Dougal, "Synergetic Control for DC-DC Boost Converter: Implementation Opt”, IEEE Trans. on Indus. Appl., Vol. 39, No. 6, pp. 1803-1813, Dallas, Nov. 2003.

\section{AUTHOR:}

Mohamed N. Harmas is with QuERE Laboratory, Electrical Engineering Department at University of Setif1, Algeria. His research interests include robust nonlinear control, power and power electronics control.

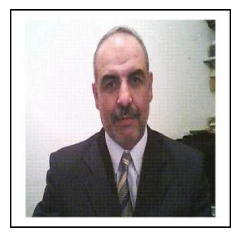

